\title{
The cellulose-binding activity of the PsB multiprotein complex is required for proper assembly of the spore coat and spore viability in Dictyostelium discoideum
}

\author{
Supriya Srinivasan, ${ }^{1}$ Katherine R. Griffiths, ${ }^{2}$ Vince McGuire, ${ }^{1}$ \\ Alan Champion, ${ }^{2}$ Keith L. Williams ${ }^{2}+$ and Stephen Alexander ${ }^{1}$
}

1 Division of Biological Sciences, University of Missouri, Columbia, MO 65211-7400, USA

2 MUCAB, Department of Biological Sciences, Macquarie University Sydney, NSW 2109, Australia

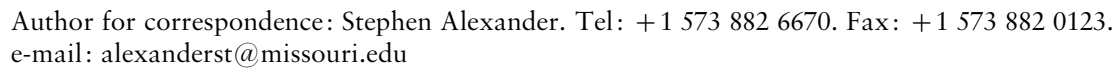

The terminal event of spore differentiation in the cellular slime mould Dictyostelium discoideum is the assembly of the spore coat, which surrounds the dormant amoeba and allows the organism to survive during extended periods of environmental stress. The spore coat is a polarized extracellular matrix composed of glycoproteins and cellulose. The process of spore coat formation begins by the regulated secretion of spore coat proteins from the prespore vesicles (PSVs). Four of the major spore coat proteins (SP96, PsB/SP85, SP70 and SP60) exist as a preassembled multiprotein complex within the PSVs. This complete complex has an endogenous cellulose-binding activity. Mutant strains lacking either the SP96 or SP70 proteins produce partial complexes that do not have cellulose-binding activity, while mutants lacking SP60 produce a partial complex that retains this activity. Using a combination of immunofluorescence microscopy and biochemical methods we now show that the lack of cellulose-binding activity in the SP96 and SP70 mutants results in abnormally assembled spore coats and spores with greatly reduced viability. In contrast, the SP60 mutant, in which the PsB complex retains its cellulosebinding activity, produces spores with apparently unaltered structure and viability. Thus, it is the loss of the cellulose-binding activity of the PsB complex, rather than the mere loss of individual spore coat proteins, that results in compromised spore coat structure. These results support the idea that the cellulose-binding activity associated with the complete PsB complex plays an active role in the assembly of the spore coat.

Keywords: protein targeting, prespore vesicle (PSV), extracellular matrix (ECM), glycoproteins, plant cell wall

\section{INTRODUCTION}

Extracellular matrices (ECMs) play important structural and instructive roles in cell differentiation and morphogenesis (Lukashev \& Werb, 1998). The cellular slime mould Dictyostelium discoideum produces unique ECMs at each different stage of development, including aggregation, slug migration and spore differentiation (Alexander, 1997). A tough spore coat ECM surrounds

†Present address: Proteome Systems Limited, Locked Bag 2073, North Ryde Sydney, NSW 1670, Australia.

Abbreviations: ECM, extracellular matrix; LPS, lower pad solution; PSV, prespore vesicle. the mature spores and is composed of equal parts of cellulose and a number of specific spore coat glycoproteins (West \& Erdos, 1990). The spore coat protects the encased dormant amoeba from a variety of harmful environmental stresses, including temperature and desiccation. This protection allows the spores to remain viable for extended periods of time in unfavourable conditions and thus provides a substantial evolutionary advantage to the organism.

The spore coat of the mature spores is a tri-lamellar, polarized structure and has been studied by electron microscopic as well as biochemical methods. The outer layer of the spore coat is composed of loosely associated 
proteins, the middle layer contains cellulose and the inner layer of the spore coat is composed of proteins that are bound covalently to the coat (Devine et al., 1982; Hemmes et al., 1972; Orlowski \& Loomis, 1979). A layer of galactose- $\mathrm{N}$-acetylgalactosamine-containing mucopolysaccharide (GPS) lies just proximal to the plasma membrane of the underlying dormant amoeba (Erdos \& West, 1989). A multiprotein complex called the PsB complex is found within the spore coat of mature spores and spans the three layers of the spore coat. The PsB complex is made up of four major glycoproteins, SP96, PsB/SP85, SP70 and SP60, which are held together by both covalent and non-covalent bonds (Watson et al., 1993). The proteins of the complex are expressed during the slug stage of development and accumulate in this preassembled form in prespore vesicles (PSVs) (Devine et al., 1983; Watson et al., 1994). At the final stage of spore differentiation, an unknown developmental signal induces the synchronous fusion of the PSVs with the plasma membrane followed by the subsequent secretion of the PsB complex, as well as other uncomplexed spore coat proteins which are stored in the PSVs. One such glycoprotein is SP75, which is not part of the preassembled PsB complex in the PSVs, but is subsequently assembled into the spore coat upon secretion (Watson et al., 1994). Analysis by immunofluorescent staining with $\mathrm{mAbs}$ has shown that the PsB glycoprotein complex becomes part of both the spore coat and the interspore matrix which can be visualized as a phase-dense material between mature spores (Watson et al., 1994).

The PsB complex has an endogenous cellulose-binding activity, a function which correlates well with its localization to the cellulose-rich spore coats (McGuire, 1996; McGuire \& Alexander, 1996). Using a series of mutants lacking different proteins of the PsB complex (Fosnaugh et al., 1994), we established the relative order of assembly of the proteins into the complex and showed that a fully assembled PsB complex is required for cellulose-binding activity (McGuire \& Alexander, 1996). Specifically, we found that SP96 was anchored to the complex through SP70, which in turn was bound to PsB. The abrogation of either SP96 or SP70 resulted in the assembly of partial complexes (containing either SP70/PsB/SP60 or SP96/PsB/SP60, respectively) which did not have cellulose-binding activity. SP60 is anchored to the complex through PsB, but interestingly, its absence has no effect on the cellulose-binding activity of the partial complex containing SP96/SP70/PsB.

The cellulose-binding activity of the PsB complex suggested that this activity may be necessary for localization and proper assembly into the spore coat. Thus, we predicted that mutants which are defective in the cellulose-binding activity $\left(\mathrm{SP}^{-} 6^{-}\right.$and $\mathrm{SP}^{-} 0^{-}$) will have an aberrant spore coat. To test this hypothesis, we examined the mutants for (1) spore coat protein localization, (2) spore coat structure and (3) viability of the mutant spores. The availability of several well characterized $\mathrm{mAbs}$ to the peptide and oligosaccharide epitopes of the different proteins of the PsB complex has enabled us to address these questions (Champion et al., 1991, 1995; Smith et al., 1989).

In this report, using a combination of immunofluorescence microscopy and biochemical methods, we show that in the $\cot A^{-}\left(\mathrm{SP}^{-}\right)$and $\cot B^{-}\left(\mathrm{SP} 70^{-}\right)$ mutant strains, the partial PsB complexes which lack cellulose-binding activity are secreted and do localize to the spore coat. This suggests that assembly of all components of the complex and the associated cellulosebinding property are not a prerequisite for its routing, secretion and localization. However, in these mutants, the structure of the spore coat is altered and results in a dramatic loss of viability of the spores compared to their wild-type parent. On the other hand, the $\cot \mathrm{C}^{-}\left(\mathrm{SP}^{-} 0^{-}\right)$ mutant in which the partial PsB complex retains its cellulose-binding activity, produces spores with apparently normal structure. Thus the proper construction and assembly of the spore coat and the viability of the spores is directly correlated with the cellulose-binding activity of the PsB multiprotein complex.

\section{METHODS}

Strains and cell culture conditions. D. discoideum cells were grown in association with Klebsiella aerogenes on slime mould medium agar plates at $22^{\circ} \mathrm{C}$ (Sussman, 1987). Spore coat protein mutant strains and the parental strain HL328 were generously provided by Bill Loomis and Danny Fuller (University of California, San Diego, USA). Table 1 summarizes the strains and their properties.

Immunofluorescence microscopy. Wild-type and mutant spores were harvested $1 \mathrm{~d}$ after the first appearances of spores. Spores were harvested and washed in lower pad solution (LPS; $20 \mathrm{mM} \mathrm{KCl}, 2.5 \mathrm{mM} \mathrm{MgCl}_{2}, 40 \mathrm{mM}$ potassium phosphate, $\mathrm{pH} 6.5$ ) containing $0.5 \mathrm{mg}$ streptomycin sulfate $\mathrm{ml}^{-1}$ and then were either treated directly for immunofluorescence microscopy or germinated before treatment (Ennis \& Sussman, 1975). Untreated or germinated spores were allowed to settle and adhere to coverslips, fixed with $3.7 \%(\mathrm{v} / \mathrm{v})$ formaldehyde in LPS, incubated for $3.5 \mathrm{~min}$ and washed for $10 \mathrm{~min}$ in LPS. Cells were permeabilized by incubating the coverslips with $0.5 \%(\mathrm{v} / \mathrm{v})$ Nonidet-P40 in LPS for $5 \mathrm{~min}$, followed by three washes for $5 \mathrm{~min}$ each in LPS. Non-specific binding sites were blocked by incubation for $20 \mathrm{~min}$ in filter-sterilized $1 \%(\mathrm{w} / \mathrm{v})$ BSA (Fraction V; Sigma) in LPS ( $80 \mu \mathrm{l}$ per coverslip). Each coverslip was then incubated for $2 \mathrm{~h}$ with $80 \mu \mathrm{l}$ of a $1: 500$ dilution of primary antibody in $1 \%$ BSA/LPS. Table 2 summarizes the antibodies used in this study. Parallel negative controls were incubated in the same solution without the primary antibody. The coverslips were drained, washed three times with LPS and incubated for one hour with $80 \mu \mathrm{l}$ of a 1:500 dilution of affinity-purified rhodamine-conjugated goatanti mouse immunoglobulin of the appropriate specificity (Cappel/Organon Teknika) in 1\% BSA/LPS. The coverslips were washed three times in LPS and were mounted onto slides (cell side down) in a solution of Airvol (Air Products) in LPS containing $50 \mathrm{mg}$ DABCO $\mathrm{ml}^{-1}$ (1,4-diazabicyclo[2.2.2]octane) to prevent fading of the fluorescence. All slides were examined and photographed with a Zeiss IM inverted microscope using a $100 \times$ Neofluar lens. At least 100 spores were examined for each treatment.

Extraction of spore coat proteins. Spores were washed sequentially in a series of four buffers to differentially extract the spore coat proteins. Each wash was repeated three times to 
Table 1. D. discoideum strains used in this study

\begin{tabular}{|lccc|}
\hline Disrupted gene(s) & Cognate protein & Strain & $\begin{array}{c}\text { Cellulose-binding } \\
\text { activity of } \\
\text { the PsB complex* }\end{array}$ \\
\hline Parental wild-type & - & HL328† & + \\
$\cot A$ & SP96 & TL55 & - \\
$\cot B$ & SP70 & TL26 & - \\
$\cot C$ & SP60 & TL52 & + \\
$\cot A, \cot B, \cot C$ & SP96, SP70, SP60 & TL56 & - \\
\hline
\end{tabular}

* McGuire \& Alexander (1996).

† Kuspa \& Loomis (1994).

‡Fosnaugh et al. (1994).

Table 2. mAbs used in this study

\begin{tabular}{|lcl|}
\hline mAb & Cognate protein & \multicolumn{1}{c|}{ Epitope } \\
\hline MUD3 & SP96 & Fucose epitope* + \\
MUD141 & SP96 & Fucose epitope* $†$ \\
MUD62 & SP96, SP75 & O-Glycosylated carbohydrate moiety†‡ \\
MUD102 & PsB & Peptide backbone $\mathbb{S}$ \\
\hline
\end{tabular}

\footnotetext{
*Voet et al. (1985).

† Champion et al. (1995).

‡Grant \& Williams (1983).

SSmith et al. (1989).
}

ensure complete extraction. Over $90 \%$ of the extractable protein was found in the first round and only the first extracts are shown in the figures. One-day-old spores $\left(1 \times 10^{7}\right)$ were collected from bacterially grown cells into $1 \mathrm{ml}$ Tris buffer (10 mM Tris/ $\mathrm{HCl}, \mathrm{pH} 8 \cdot 0)$, with the freshly added protease inhibitors, $100 \mu \mathrm{M}$ AEBSF [4-(2-aminoethyl)benzenesulfonyl fluoride $/ \mathrm{HCl}$; Boehringer Mannheim] and $3 \mu \mathrm{M}$ E64c (transepoxysuccinyl-L-leucylamido-3-methylbutane; Sigma). The spores were pelleted for $2 \mathrm{~min}$ in a microcentrifuge at 14000 r.p.m. and the supernatant (wash 1) was kept at $4{ }^{\circ} \mathrm{C}$ for analysis. The spores were then resuspended in $1 \mathrm{ml}$ Tris buffer containing $1 \%$ SDS (Tris/SDS), incubated for $5 \mathrm{~min}$, pelleted and the supernatant (wash 2) was kept. The spores were then boiled for $5 \mathrm{~min}$ in Tris/SDS before pelleting (wash 3) and then finally boiled for $5 \mathrm{~min}$ in Tris/SDS buffer containing $5 \%$ (v/v) 2-mercaptoethanol (wash 4). For Western blot analysis, $20 \mu \mathrm{l}$ of each wash was loaded per lane, equivalent to extracts from $2 \times 10^{5}$ spores.

Spore viability assays. Strains were mass-plated in association with Klebsiella aerogenes. Spores of each strain were tested for viability by two methods, according to a procedure adapted from Richardson \& Loomis (1992). In the first test, spores were allowed to remain on stalks on slime mould medium plates at $22{ }^{\circ} \mathrm{C}$ for the duration of the experiment $(30 \mathrm{~d})$. Spores were collected every $5 \mathrm{~d}$, resuspended in $10 \mathrm{mM}$ EDTA at $1 \times 10^{5} \mathrm{ml}^{-1}$ and incubated at $37^{\circ} \mathrm{C}$ for $30 \mathrm{~min}$. Duplicate serial dilutions in Bonner's standard solution/5 mM EDTA were plated on slime mould medium plates in association with $K$. aerogenes. The number of plaques formed was counted and spore viability was expressed as the percentage of the number of plaques seen on day 1 of the experiment. In the second test, on day 1 spores were collected and resuspended to $1 \times 10^{8} \mathrm{ml}^{-1}$ in $50 \mathrm{mM}$ sodium phosphate, $\mathrm{pH} 6.4$, with $1 \%$ antibiotic/antimycotic (Gibco-BRL). The spore suspensions were then shaken at $22{ }^{\circ} \mathrm{C}$ for the duration of the experiment (30 d). Viability was tested every $5 \mathrm{~d}$ as described above. Note that for the $\cot A B C^{-}$strain, owing to a severe loss in viability, it was not possible to conduct the experiment for longer than $20 \mathrm{~d}$.

\section{RESULTS}

\section{Cot mutant strains lacking cellulose-binding activity have altered spore coats}

To test whether mutant strains lacking cellulose-binding activity had altered spore coat structure, we analysed the spore coats in wild-type and four cot mutant strains. The data are summarized schematically in Fig. 4.

Immunofluorescence microscopy. The localization of the spore coat components in the cot gene deletion mutant strains (Table 1) was examined in intact and germinated spores by immunofluorescence microscopy, using either the MUD3 or MUD102 mAbs, which recognize the SP96 and PsB proteins, respectively (Table 2). No fluorescence was seen when either untreated or germinated spores were incubated with secondary antibody alone (Fig. 1A-E, panels a).

Parental strain HL328. The localization of SP96 and PsB to the spore coat layers in the parental strain HL328 was identical to that previously seen in the wild-type 
A
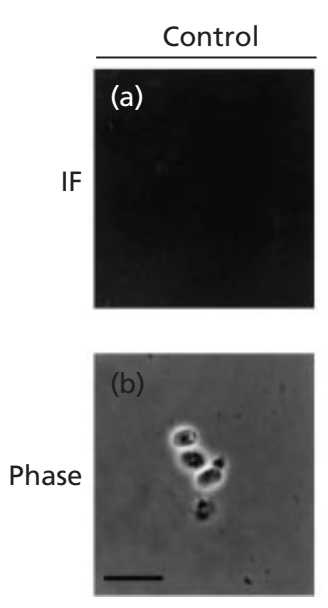

B
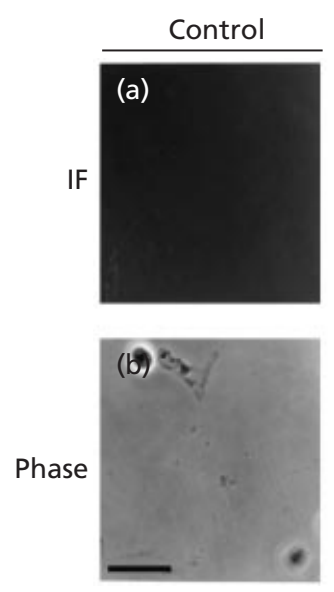

C
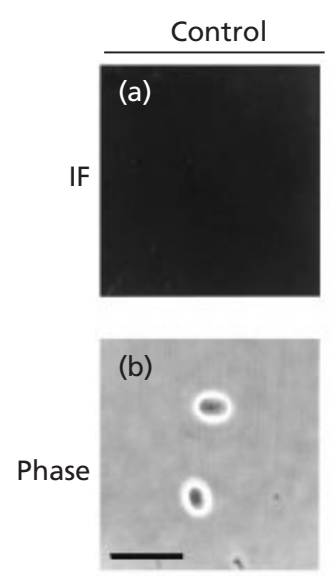

MUD3 (anti-SP96)
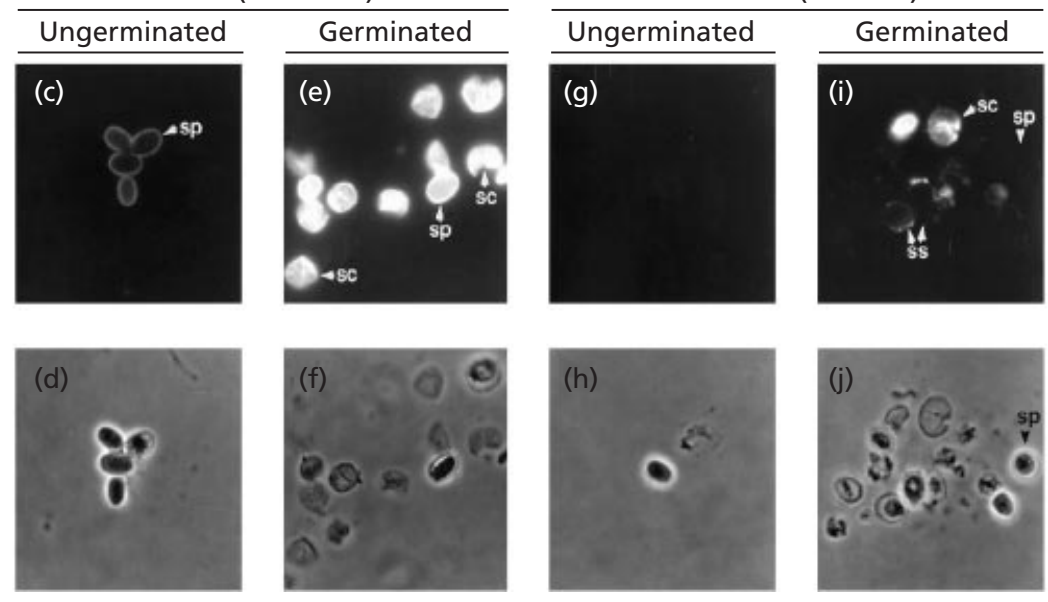

MUD102 (anti-PsB)
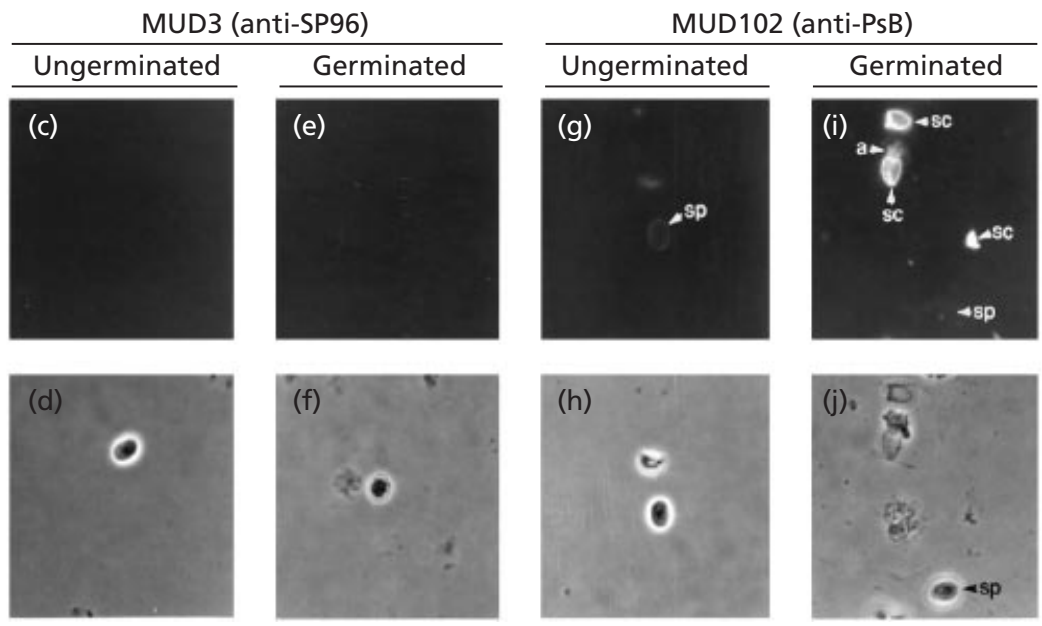

MUD102 (anti-PsB)

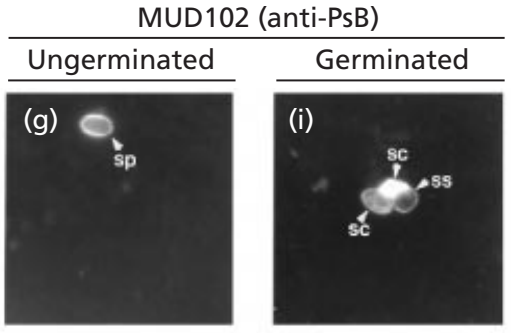

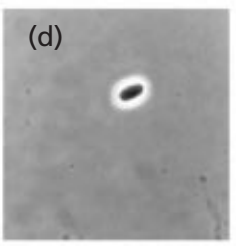

MUD3 (anti-SP96)
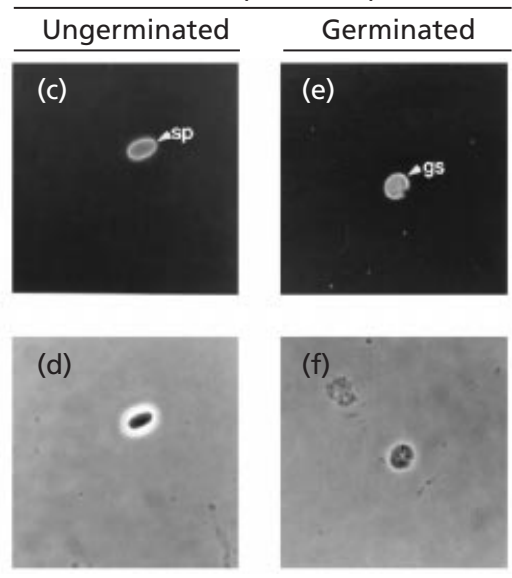
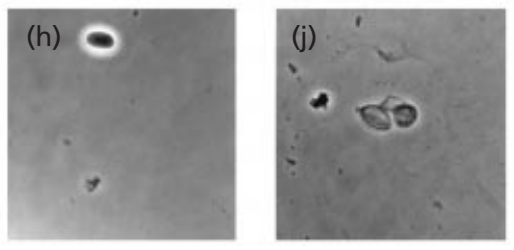

Fig. 1. For legend see facing page.

strain WS380B (Richardson \& Loomis, 1992; Watson et al., 1994). Intact spores fluoresced strongly when labelled with MUD3, demonstrating that SP96 was exposed to the outer layer of the spore coat (Fig. 1A, c).
Stronger MUD3 fluorescence was observed from germinated spores, including empty spore coats, confirming that SP96 remains associated with the coat after germination (Fig. 1A, e). The intact spores did not stain 
D
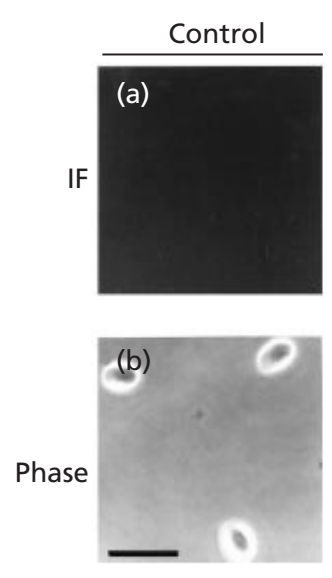

$\mathrm{E}$
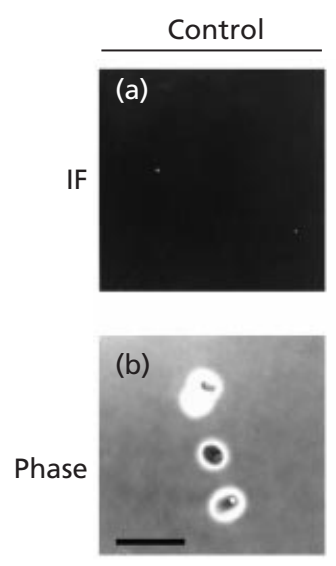

MUD3 (anti-SP96)
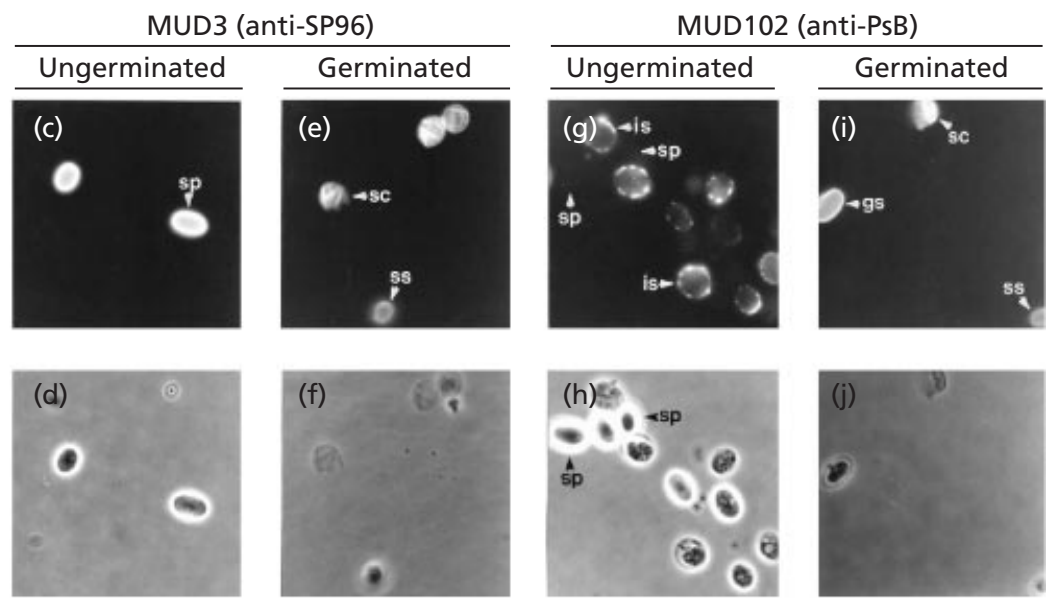

MUD102 (anti-PsB)

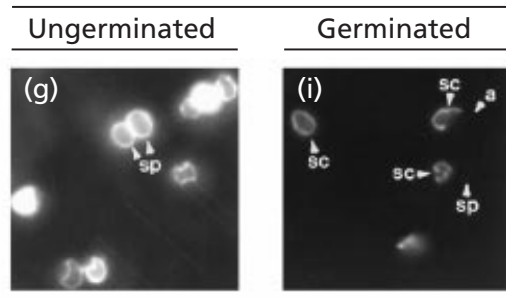

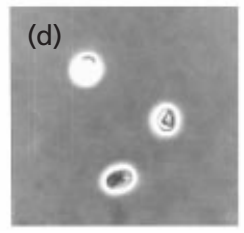

MUD3 (anti-SP96)
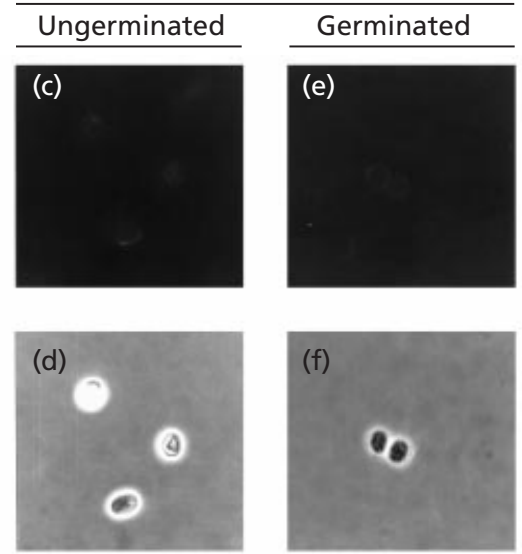
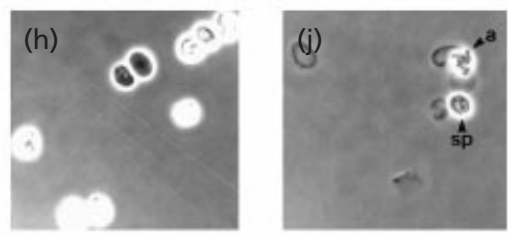

Fig. 1. Immunofluorescent analysis of parental HL328 and cot mutant spores. Spores were harvested and immunostained with MUD3 (anti-SP96) or MUD102 (anti-PsB) after fixation on coverslips. Immunofluorescent (IF) images are shown on the top rows $(a, c, e, g, i)$ and the corresponding phase-contrast (phase) images are shown on the bottom rows (b, d, f, h, j). $(a, b)$ Spores treated with the rhodamine-conjugated secondary antibody alone. (c, d) Ungerminated and (e, $f$ ) germinated spores treated with MUD3. $(\mathrm{g}, \mathrm{h})$ Ungerminated and $(\mathrm{i}, \mathrm{j})$ germinated spores treated with MUD102. Treatments are indicated above the panels. A, Parental HL328 spores; B, $\left.\cot A^{-}(\mathrm{SP96})^{-}\right)$mutant spores; $\mathrm{C}_{1} \cot B^{-}\left(\mathrm{SP70} 0^{-}\right)$

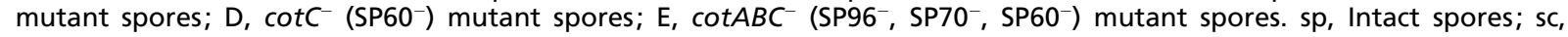
empty spore coats; ss, swollen spores; a, amoebae; gs, opened germinated spores; is, immature spores. Bars, $10 \mu \mathrm{m}$. Note that no MUD3 staining is visible in $B$ and $E$, as the SP96 protein that this antibody recognizes is missing in $\cot A^{-}$and $\cot A B C^{-}$mutant strains.

when labelled with MUD102 (Fig. 1A, g), but germinated spores and empty spore coats fluoresced strongly, confirming that PsB is exposed on the inner layer of the spore coat and is only available to its cognate $\mathrm{mAb}$ when the inner layer of the spore coat is exposed after germination (Fig. 1A, i).

$\cot A$ deletion mutant (SP96). The $\cot A^{-}$strain lacks the SP96 protein. As expected, no labelling with MUD3 was observed with either untreated (Fig. 1B, c) or germinated spores (Fig. 1B, e). In contrast to the wildtype parental strain, some MUD102 fluorescence was visible in intact, untreated spores (Fig. 1B, g) and stronger MUD102 fluorescence was seen in germinated spores (Fig. 1B, i). These data indicate that the partial
PsB complex still localizes to the spore coat in the absence of SP96. However, the PsB protein, which normally localizes to the inner layer of the coat is now exposed and available for recognition by MUD102 (Fig. $4 b)$.

cotB deletion mutant (SP70). The $\cot B^{-}$strain lacks the SP70 protein. Although there are no mAbs available that specifically recognize this protein, the fate of other coat proteins can be followed in this strain. Previously, it has been shown that this protein mediates the association of SP96 $(\cot A)$ with the PsB protein because in cells lacking SP70, SP96 is no longer associated with the complex (McGuire \& Alexander, 1996). When $\cot B^{-}$ spores were stained with MUD3, strong fluorescence 
could be seen on the surface of both ungerminated (Fig. 1C, c) and germinated spores (Fig. 1C, e). These results indicate that SP96 is localized to the spore coat even when not in association with the rest of the PsB complex prior to secretion. The spores labelled with the MUD102 fluoresced brightly, in both intact (Fig. 1C, g) and germinated spores (Fig. 1C, i), demonstrating a clearly altered spore coat structure in mutants lacking SP70 where PsB is now available for binding to MUD102 in intact spores (Fig. 4c).

cotC deletion mutant (SP60). The $\cot \mathrm{C}^{-}$strain lacks the SP60 protein. The absence of this protein does not affect the assembly of the rest of the PsB complex or its cellulose-binding activity (McGuire \& Alexander, 1996). As expected, MUD3 fluorescence was observed in both the untreated (Fig. 1D, c) and germinated spores (Fig. 1D, e), indicating that the localization of SP96 was not affected by the loss of SP60. MUD102 labelling was not visible on intact spores (Fig. 1D, g) but was bright on germinated spores (Fig. 1D, i), indicating that spores lacking SP60 have a normal spore coat structure (Fig. $4 \mathrm{~d})$. Interestingly, ungerminated immature spores in this sample did show some fluorescence with MUD102 (Fig. $1 \mathrm{D}, \mathrm{g})$, presumably because the coat is not yet completely matured, and suggests that development in this strain may be somewhat slower than the wild-type.

$\cot A B C$ deletion mutant (SP96, SP70 and SP60). The $\cot A B C^{-}$strain lacks the SP96, SP70 and SP60 proteins. When spores from this strain were labelled with MUD3, no fluorescent signal was seen on either untreated (Fig. 1E, c) or germinated spores (Fig. 1E, e). The MUD102 fluorescence was very bright on intact spores (Fig. 1E, g), though somewhat less bright on germinated spores (Fig. 1E, i). This labelling confirms that the PsB protein is still present and localized to the spore coat, but that the structure of the spore coat is greatly altered (compare Figs 1A, g and 1E, g).

Flow cytometry was performed on unfixed spores to confirm the results obtained from the immunofluorescence experiments, thus eliminating the possibility of fixation artefacts. MUD3 staining showed that SP96 was present on the outer layer of the spore coat in wild-type, $\cot B^{-}$and $\cot C^{-}$spores and, as expected, was absent in spores from $\cot A^{-}$and $\cot A B C^{-}$strains. PsB was exposed to the outside of the spores in the $\cot A^{-}$, $\cot B^{-}$and $\cot A B C^{-}$strains, but not in the wild-type and $\cot C^{-}$spores (data not shown).

Biochemical extractions. The immunofluorescent analysis showed that the structure of the spore coat was altered in mutants with partial PSB complexes which lack cellulose-binding activity. To determine whether these changes in coat structure were reflected at the biochemical level, we examined the structure of the spore coat in the parental strain HL328 and the four cot mutant strains by a series of extractions. The extracted proteins were separated by SDS-PAGE and analysed by Western blotting, using the mAbs MUD62, MUD141, MUD3 (all recognizing SP96) and MUD102 (recognizing PsB) (Table 2 and Fig. 2). The biochemical data confirm the results from immunofluorescence experiments and show that spores from the $\cot A^{-}, \cot B^{-}$and $\cot A B C^{-}$ strains are altered, and that the spores from the $\cot C^{-}$ strain are similar to the wild-type.

Parental strain HL328. The extractions of the parental strain produced results identical to those seen in a previous large-scale extraction of spores from the wildtype strain WS380B as assayed by Western analysis with mAbs MUD62 and MUD102 (Watson et al., 1994). SP96 is found in all extracts when tested with MUD62 (Watson et al., 1994), with the majority in the fraction extracted in hot SDS (Fig. 2a). When the fractions were tested with the mAbs MUD3 or MUD141, SP96 was detectable only in fraction 3. This is most likely due to a considerably lower affinity of these antibodies for SP96 compared to MUD62. The PsB glycoprotein was extracted in all washes, although it was most predominant in wash 4 (Fig. 2a). This result indicates that the majority of PsB is covalently attached to the spore coat. Washes 1 and 2 remove the interspore matrix between spores and loosely associated proteins, respectively. The PsB found in washes 1 and 2 indicates the presence of the PsB complex in the interspore matrix, as observed previously (Watson et al., 1994).

It is important to note that the majority of SP96 (wash 3) does not co-fractionate with the majority of the PsB protein (wash 4), although SP96 has been shown to be covalently linked to the PsB complex through disulfide bridges (Watson et al., 1993). This observation suggests that SP96 is expressed in large excess over the other proteins in the PsB complex. This idea is supported by experiments in which labelled cell extracts that were depleted of the PsB multiprotein complex by sequential immunoprecipitations with MUD102 still contained free SP96, as demonstrated by immunoprecipitation with MUD62 (unpublished).

cotA deletion mutant (SP96). SP96, assayed by MUD62, MUD3 or MUD141, is missing from all the fractions (Fig. 2b). PsB was found only in fraction 4 from this strain, confirming that in the absence of SP96, the partial PsB complex can still localize and be covalently bound to the spore coat. However, the distribution of PsB to the interspore matrix appears to depend on the presence of SP96.

cotB deletion mutant (SP70). In the absence of SP70, SP96 does not associate with the PsB complex (McGuire \& Alexander, 1996). The immunofluorescence data indicated that SP96 is still incorporated into the spore coat in this mutant. However, staining with MUD141 and MUD3 show that the distribution of SP96 is altered in this mutant (Fig. 2c). There was an increase in SP96 in fractions 1 and 2 in contrast to the parental strain HL328 (compare Fig. 2a and 2c), because when SP70 is absent, SP96 is not bound to the PsB complex and therefore is extracted under milder conditions (wash 1). The PsB distribution was virtually identical to that observed in the parental strain.

cotC deletion mutant (SP60). The distribution of SP96 in this strain is identical to the parental strain with almost 

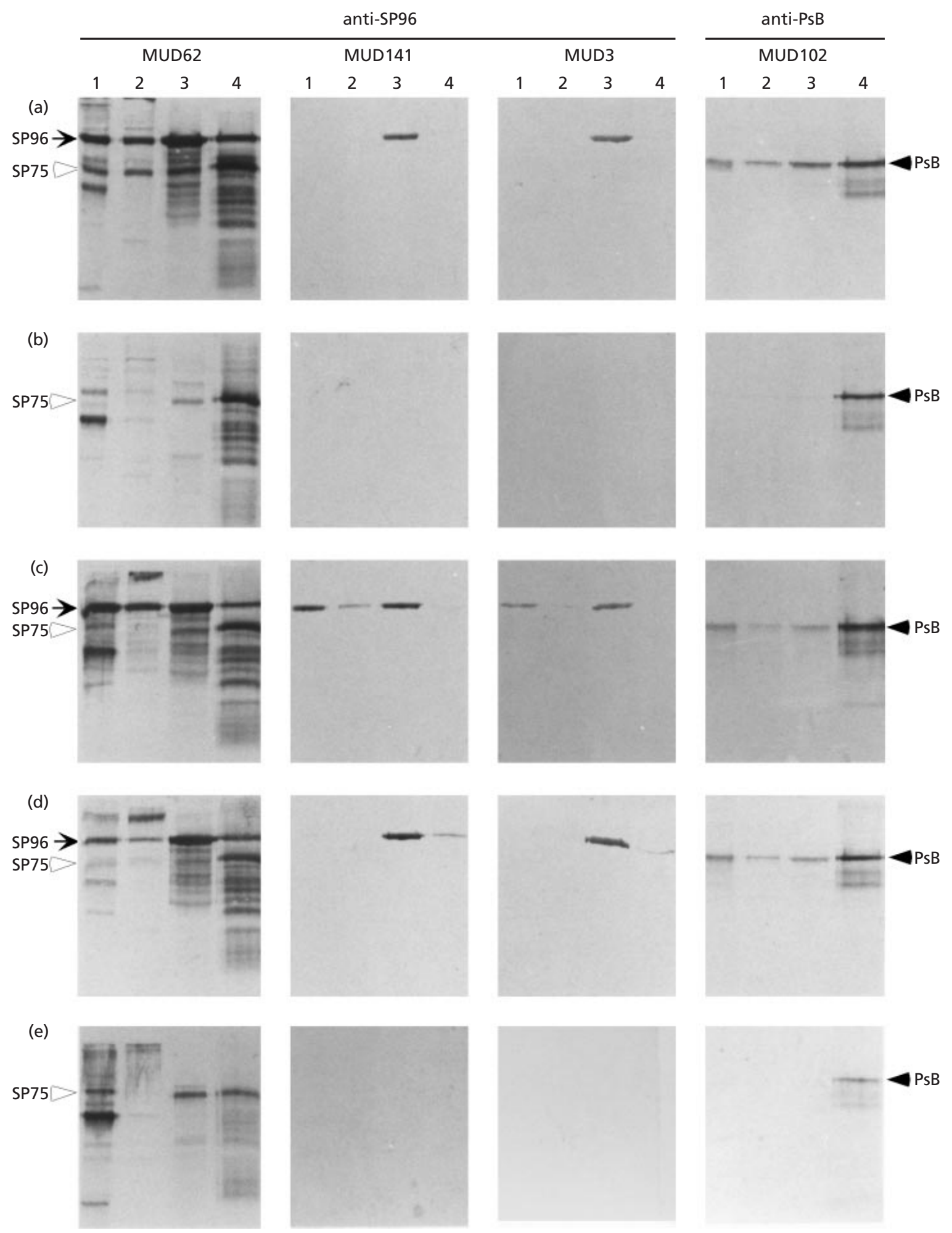

Fig. 2. Biochemical analysis of parental strain $H L 328$ (a) and the $\cot$ mutant strains $\cot A^{-}$(b), $\cot B^{-}$(c), $\cot C^{-}(d)$ and $\cot A B C^{-}(\mathrm{e})$. Spores $\left(1 \times 10^{7}\right)$ from each of the five strains were collected and subjected to the series of extractions described in Methods. Lanes: 1, wash 1, Tris buffer; 2, wash 2, cold Tris/SDS buffer; 3, wash 3, hot Tris/SDS buffer; 4, wash 4, hot Tris/SDS buffer containing 2-mercaptoethanol. Lanes 1-4 on each blot represent the extracts from $2 \times 10^{5}$ spores from each of the four extractions. The blots were probed with MUD62, MUD3, MUD141 or MUD102 as indicated (Table 2). 


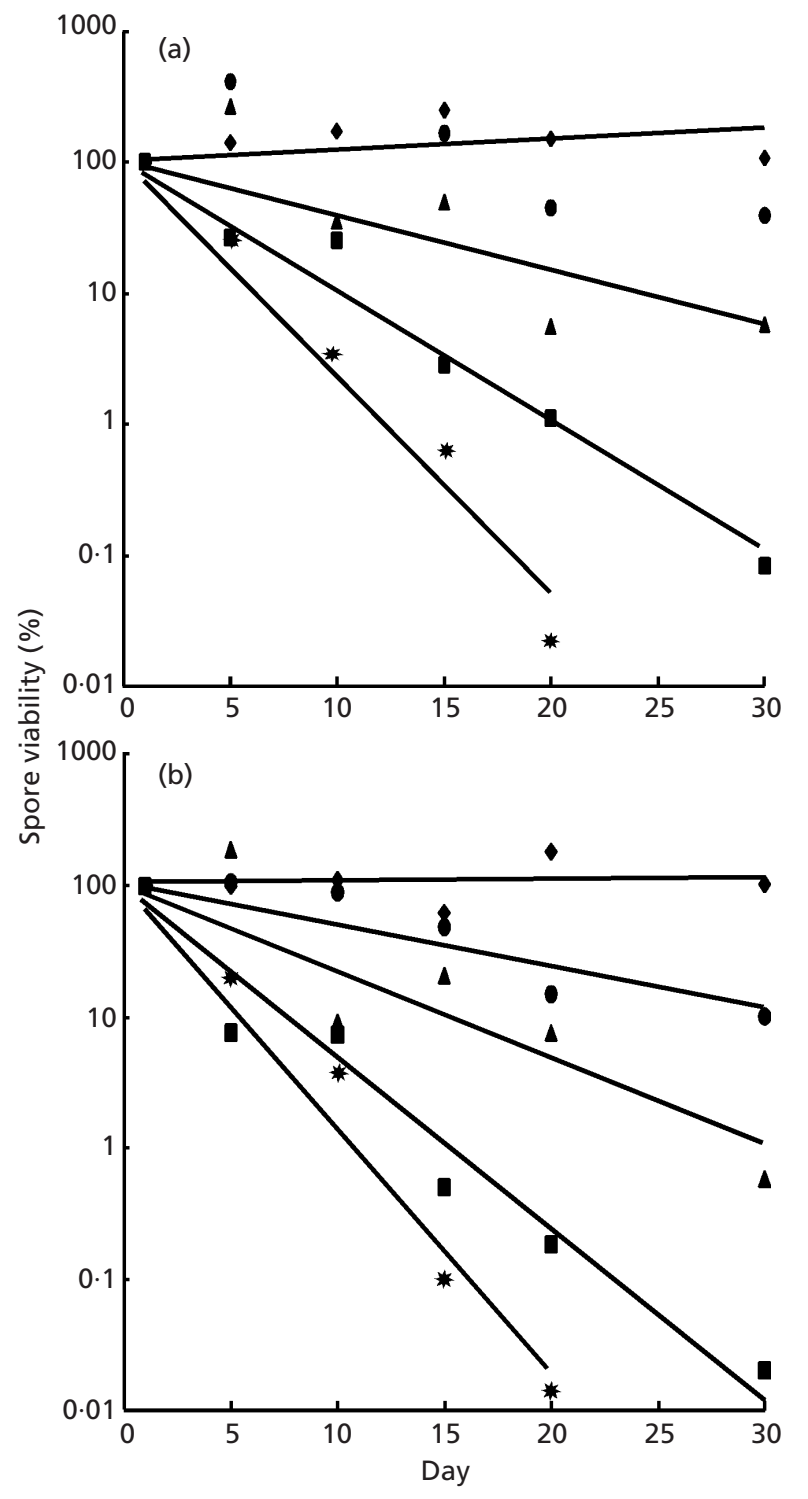

Fig. 3. Spore viability assays. (a) For each strain, spores were allowed to remain on stalks at $22{ }^{\circ} \mathrm{C}$ for the duration of the experiment $(30 \mathrm{~d})$. (b) For each strain, spores were harvested on day 1 and were shaken at a density of $1 \times 10^{8} \mathrm{ml}^{-1}$ in $10 \mathrm{mM}$ sodium phosphate buffer, $\mathrm{pH} 6 \cdot 5$, at $22{ }^{\circ} \mathrm{C}$ for $30 \mathrm{~d}$. Every $5 \mathrm{~d}$, spores were collected and resuspended to $1 \times 10^{5} \mathrm{ml}^{-1}$ in $10 \mathrm{mM}$ EDTA, incubated at $37^{\circ} \mathrm{C}$ for $30 \mathrm{~min}$ and duplicate serial dilutions were plated for viability as described in Methods. Spore viability is expressed as a percentage of the number of plaques seen on day 1 of the experiment. $\bullet, H L 328 ; \square, \cot A^{-}$; $\Delta, \cot B^{-} ; 0, \cot C^{-} ; *, \cot A B C^{-}$. Each point is a mean of duplicate determinations.

all of SP96 in fraction 3 (Fig. 2d). The pattern of MUD102 labelling was also identical to the parental strain.

$\cot A B C$ deletion mutant. The results with the triple mutant are identical to those seen with the $\cot A^{-}$strain, which also lacks SP96 (Fig. 2e). Again, only fraction 4 contains the PsB protein.

The SP75 glycoprotein is found in the spore coat of mature spores, but is not a component of the preassembled PsB complex (Watson et al., 1994; West et al., 1996). It is recognized by the mAb MUD62 and in all strains tested, SP75 is extracted predominantly in the fourth wash (Fig. 2a-e). It is therefore cross-linked normally into the coat in the different cot mutants.

\section{Spores with partial PsB complexes have lower viability}

The immunofluorescence and biochemical extraction experiments described so far demonstrate a change in the structure of the spore coat in the $\cot A^{-}, \cot B^{-}$and $\cot A B C^{-}$strains. No alteration was seen with the $\cot C^{-}$ strain when compared to the parental strain. These data are in accord with our observation that only the $\cot \mathrm{C}^{-}$ strain produces a partial PsB multiprotein complex that still retains cellulose-binding activity (McGuire \& Alexander, 1996). Since proper spore coat assembly and structure are crucial to the survival of the organism, we predicted that there would be a loss of spore viability in mutants where the spore coats were altered.

To test this, spores from the wild-type parent and the four mutant strains were tested for their viability over a period of $30 \mathrm{~d}$. In the first test, spores were allowed to remain on stalks at $22{ }^{\circ} \mathrm{C}$ for the duration of the experiment and their viability was tested every $5 \mathrm{~d}$ as described in Methods. The data are presented in Fig. $3(\mathrm{a})$. The wild-type parent showed no loss of viability over $30 \mathrm{~d}$. The most dramatic effects were seen in the $\cot A^{-}$and $\cot A B C^{-}$strains, where a 1300 - and 3750 -fold loss in viability was observed, respectively. The $\cot B^{-}$ strain showed an 18 -fold reduction in viability. However, only a threefold decrease in viability was seen in the $\cot \mathrm{C}^{-}$mutant spores.

In the second test, spores were submerged in buffer at a density of $1 \times 10^{8} \mathrm{ml}^{-1}$ on day 1 , were kept shaking at $22{ }^{\circ} \mathrm{C}$ for $30 \mathrm{~d}$ and were sampled every $5 \mathrm{~d}$. Although the wild-type parent again showed no loss of viability over the period tested, a more severe effect was seen in all mutant strains, as shown in Fig. 3(b). The $\cot A^{-}$and the $\cot A B C^{-}$strains showed a 5000- and 8500 -fold loss in viability, respectively. The $\cot B^{-}$strain showed an 180 -fold reduction in viability and again the $\cot \mathrm{C}^{-}$strain showed a much lower 10-fold reduction in viability.

\section{DISCUSSION}

The assembly of the spore coat is the ultimate step in the development of $D$. discoideum (Srinivasan et al., 2000). Proper construction of this complex ECM affords the enclosed dormant amoeba protection from the environment. Previous biochemical and transmission electron microscopic studies have defined several spore coat components, including glycoproteins and cellulose, which are assembled into a highly organized, polar, trilamellar structure (Devine et al., 1982; Hemmes et al., 1972; West \& Erdos, 1990). However, scanning electron microscopic techniques have revealed little more about the fine structure and mechanism of assembly of the coat (Hohl \& Hamamoto, 1969; Muhlethaler, 1956). Four of 
(a)

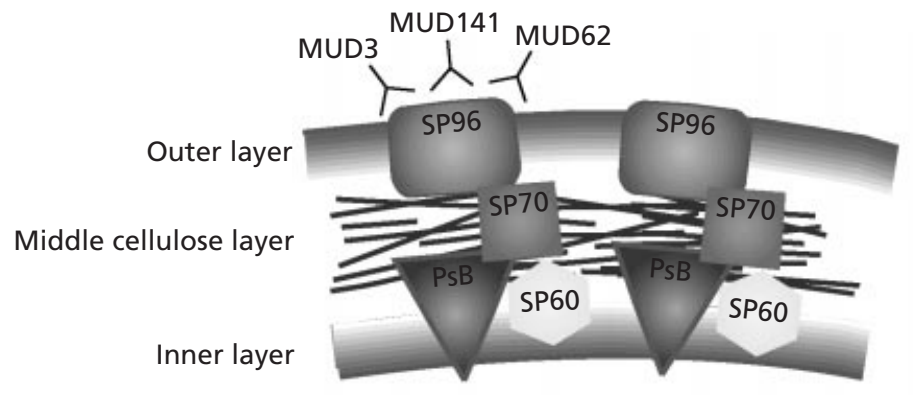

(c)

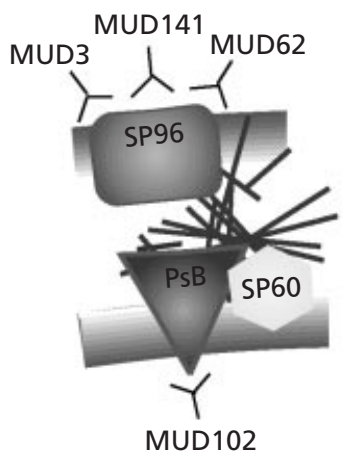

MUD102 (b)
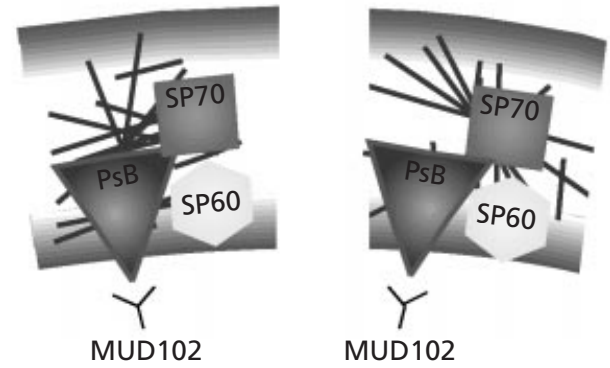

(d)
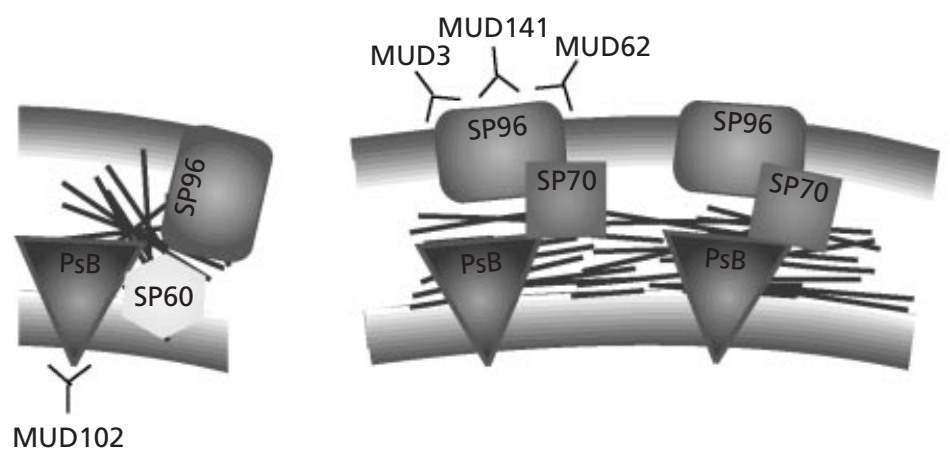

Fig. 4. Model for the role of the PsB multiprotein complex cellulose-binding activity in the assembly of the $D$. discoideum spore coat. (a) Parental strain, HL328. The complete PsB complex has cellulose-binding activity which results in a properly organized impermeable spore coat. Only proteins of the PsB complex which are on the outside of the intact spores are accessible to antibodies, giving rise to the staining patterns seen in Fig. 1A. PsB is on the inside of the coat and the MUD102 antibody cannot access PsB in ungerminated spores, thus there is no staining. (b) $\cot A^{-}$mutant. The partial PsB complex does not have cellulose-binding activity and results in an improperly assembled coat which is now permeable to the antibody MUD102 and results in the staining pattern seen in Fig. 1B. (c) cotB mutant. The partial PsB complex does not have cellulose-binding activity and results in an improperly assembled coat which is now permeable to the antibody MUD102 and results in the staining pattern seen in Fig. 1C. Note that the free SP96 is localized in the coat. (d) cotC mutant. The partial PSB complex has normal cellulose-binding activity which results in an intact spore which is stained with antibodies (Fig. 1D) like the wild-type parent. We propose that the cellulose fibrils are disorganized in the mutants lacking cellulose-binding activity and this results in spores with compromised viability. Only members of the PsB complex are drawn. Other proteins, such as SP75 and SpiA, which are known to reside in the coat are not shown in this model.

the major spore coat proteins (SP96, PsB, SP70 and SP60) are preassembled into the PsB multiprotein complex which is stored within the PSVs (Devine et al., 1983; Watson et al., 1994). The PsB complex has endogenous cellulose-binding activity and the results suggested that the assembly of the PsB complex subunits is a prerequisite for the cellulose-binding activity. Indeed, none of the individual proteins have the classical cellulose-binding motifs seen in the cellulase enzymes. Mutants lacking either the SP96 or SP70 proteins of the complex, due to the deletion of the cognate genes, form incomplete complexes which do not retain cellulosebinding activity, while mutants lacking SP60 retain this activity (McGuire \& Alexander, 1996). Since the PsB complex localizes to the cellulose-rich spore coat, these data led to the hypothesis that the cellulose-binding activity of the PsB complex plays an active role in the normal development of the spore coat. The data in this paper clearly demonstrate that there is a direct cor- relation between the cellulose-binding activity of the PsB complex, the structural integrity of the spore coat and the viability of the spores.

Using immunofluorescence microscopy and biochemical extractions, we first showed that partial PsB complexes and free SP96 do localize to the coat even when they do not have cellulose-binding activity. However, in the cases of the $\cot A^{-}, \cot B^{-}$and $\cot A B C^{-}$strains, the coats are improperly assembled. The aberrant antibody staining of the mutant spores is due to an increased porosity of the spore coat or incorrect orientation of the PsB complex within the coat, or more likely a combination of the two factors. In contrast, deletion of the SP60 protein alone in the $\cot \mathrm{C}^{-}$strain does not alter the structure of the coat (Fig. 4). Thus, it is the loss of the cellulose-binding activity of the PsB complex, rather than the mere loss of individual spore coat proteins, that results in compromised spore coat structure. 
Examination of spore viability confirmed the correlation between cellulose-binding activity and normal spore coat assembly and provided a quantitative measure of the effect. Although loss of SP60 results in no apparent difference in spore coat structure, a small but measurable reduction in viability was observed (3-10-fold). This indicates that SP60 is an important structural component of the spore coat whose presence is essential for normal cell survival, even though it is not required for the cellulose-binding activity of the PsB complex. Our results are in disagreement with findings reported by Fosnaugh et al. (1994) who claimed that there was no compromised viability of spores from any of the spore coat protein mutants, although they did show an increased permeability to the lectin ricin A.

It has recently been shown that the C-terminal half of recombinant PsB protein binds cellulose in vitro (Zhang et al., 1998, 1999). The present study strongly supports our earlier work showing that PsB complexes that lack SP96 or SP70, but that do have the PsB protein, do not bind cellulose (McGuire \& Alexander, 1996). Thus, we suggest that the proper positioning of PsB within the fully assembled multiprotein complex allows the correct exposure of the PsB protein cellulose-binding site to cellulose in situ. In mutant strains lacking either the SP96 or SP70 proteins, the cellulose-binding site of PsB would not be available. Presumably, this idea can be tested when all the PsB complex proteins are expressed in vitro, although it must be remembered that each of these proteins is heavily glycosylated with specific oligosaccharides (Champion et al., 1995).

Overall, this and earlier studies present a picture where assembly of the PsB complex proteins results in a multiprotein complex with a specific cellulose-binding activity. Neither the complete assembly of the complex, nor the resulting binding activity is required for secretion and localization into the spore coat. However, the cellulose-binding activity is required for proper assembly and organization of the spore coat, and for the subsequent viability of the spores. Under natural conditions this reduction in viability would dramatically affect their survival. We suggest that the primary function for the cellulose-binding activity of the PsB complex is to organize the cellulose fibrils so that the spore coat is impermeable and resistant to environmental stress (Fig. 4). Other proteins, such as SP60 and SpiA (Richardson \& Loomis, 1992), which do not affect cellulose binding, also play important structural roles in the formation and maintenance of the spore coat ECM.

Nothing is known about how cellulose synthesis is coordinated with its deposition in the spore coat at terminal morphogenesis. The cellulose synthase gene in D. discoideum has recently been cloned and is expected to reveal information about cellulose deposition and assembly, although its localization within the cell is not yet known (Blanton et al., 2000). It remains to be shown how the timing of cellulose synthesis in the prespore cells is co-ordinated with the secretion of the contents of the PSVs. Earlier work from West \& Erdos (1990) suggests that spore coat protein secretion occurs before cellulose deposition and this agrees well with the conclusions of this study, which shows that the cellulosebinding activity of the PsB complex is required for the normal assembly of the spore coat.

The structure of the D. discoideum spore coat is reminiscent of plant cell walls, in that both are complex matrices composed of glycoproteins, polysaccharides and cellulose. The genesis of plant cell walls is intimately linked to the growth and development of plant cells and tissues (Delmer, 1999). Glycoproteins from cell walls of angiosperms can also be extracted under different conditions. Loosely associated proteins can be washed off with buffer, proteins bound by ionic interactions can be removed by salt washes and covalently bound proteins are extracted under strong reducing conditions (McNeil et al., 1984). During cell wall formation, cellulose is synthesized and extruded across the plasma membrane. The cellulose microfibrils are thought to self-assemble in the extracellular space, although the direction of cellulose deposition may be guided by microtubules lying adjacent to the membrane (Cosgrove, 1997). Yet, the detailed mechanisms by which plant cell wall structures are assembled are largely unknown and it is possible that there are cellulose-binding proteins that play a role in guiding this process, similar to that occurring in D. discoideum spore coats.

Recently, the PSVs, which contain most of the components of the future spore coat have been purified from different stages of development (Srinivasan et al., 1999). This has allowed us to begin an exhaustive characterization of the PSV contents by proteomics (Yan et al., 1997). An analysis of the protein content of the PSVs will reveal the presence of additional structural proteins of the spore coat, as well as enzymes which may be required for coat assembly and formation, such as protein disulfide isomerases. Such analyses are expected to increase our understanding of how the $D$. discoideum spore coat is assembled with its precise polarity and organization at terminal differentiation.

\section{ACKNOWLEDGEMENTS}

This work was partially supported by a University of Missouri Research Board Grant RB97-044. S. A. is the recipient of an American Cancer Society Faculty Research Award (FRA 448). This work was presented as partial fulfilment of the requirements for the PhD degree to S.S. and V.M. at the University of Missouri. K.L.W. acknowledges funding in support of this work by the Australian Research Council. K.R.G. was supported by a British Science and Engineering Research Council Postgraduate scholarship. Special thanks to Hannah Alexander for untiring help with this manuscript.

\section{REFERENCES}

Alexander, S. (1997). Developmental regulation and function of glycoproteins in Dictyostelium discoideum. In Dictyostelium: a Model System for Cell and Developmental Biology, pp. 349-362. Edited by Y. Maeda, K. Inouye \& I. Takeuchi. Tokyo: Universal Academy Press. 
Blanton, R. L., Fuller, D., Iranfar, N., Grimson, M. J. \& Loomis, W. F. (2000). The cellulose synthase gene of Dictyostelium. Proc Natl Acad Sci U S A 97, 2391-2396.

Champion, A., Gooley, A. A., Callaghan, M., Carrin, M. I., Bernstein, R. L., Smith, E. \& Williams, K. L. (1991). Immunodominant carbohydrate determinants in the multicellular stages of Dictyostelium discoideum. J Gen Microbiol 137, 2431-2438.

Champion, A., Griffiths, K., Gooley, A. A., Gonzalez, B. Y., Gritzali, M., West, C. M. \& Williams, K. L. (1995). Immunochemical, genetic and morphological comparison of fucosylation mutants of Dictyostelium discoideum. Microbiology 141, 785-797.

Cosgrove, D. J. (1997). Assembly and enlargement of the primary cell wall in plants. Annu Rev Cell Dev Biol 13, 171-201.

Delmer, D. P. (1999). Cellulose biosynthesis: Exciting times for a difficult field of study. Annu Rev Plant Physiol Plant Mol Biol 50, 245-276.

Devine, K. M., Morrissey, J. H. \& Loomis, W. F. (1982). Differential synthesis of spore coat proteins in prespore and prestalk cells of Dictyostelium. Proc Natl Acad Sci U S A 79, 7361-7365.

Devine, K. M., Bergmann, J. E. \& Loomis, W. F. (1983). Spore coat proteins of Dictyostelium discoideum are packaged in prespore vesicles. Dev Biol 99, 437-446.

Ennis, H. L. \& Sussman, M. (1975). Mutants of Dictyostelium discoideum defective in spore germination. J Bacteriol 124, 62-64.

Erdos, G. W. \& West, C. M. (1989). Formation and organization of the spore coat of Dictyostelium discoideum. Exp Mycol 13, 169-182.

Fosnaugh, K. L., Fuller, D. \& Loomis, W. F. (1994). Structural roles of the spore coat proteins in Dictyostelium discoideum. Dev Biol 166, 823-825.

Grant, W. N. \& Williams, K. L. (1983). Monoclonal antibody characterization of slime sheath: the extracellular matrix of Dictyostelium discoideum. EMBO J 2, 935-940.

Hemmes, D. E., Kojima-Buddenhagen, E. S. \& Hohl, H. R. (1972). Structural and enzymatic analysis of the spore wall layers in Dictyostelium discoideum. J Ultrastruct Res 41, 406-417.

Hohl, H. \& Hamamoto, S. (1969). Ultrastructure of spore differentiation in Dictyostelium: the prespore vacuole. J Ultrastruct Res 26, 442-453.

Kuspa, A. \& Loomis, W. F. (1994). Transformation of Dictyostelium: Gene disruption, insertional mutagenesis and promoter traps. Methods Mol Genet 3, 3-21.

Lukashev, M. E. \& Werb, Z. (1998). ECM signalling: orchestrating cell behaviour and misbehaviour. Trends Cell Biol 8, 437-441.

McGuire, V. (1996). Assembly and function of the PsB multiprotein complex during spore differentiation in Dictyostelium discoideum. PhD thesis, University of Missouri, USA.

McGuire, V. \& Alexander, S. (1996). PsB multiprotein complex of Dictyostelium discoideum: Demonstration of cellulose binding activity and order of protein subunit assembly. J Biol Chem 271, 14596-14603.
McNeil, M., Darvill, A. G., Fry, S. C. \& Albersheim, P. (1984). Structure and function of the primary cell walls of plants. Annu Rev Biochem 53, 625-663.

Muhlethaler, K. (1956). Electron microscopic study of the slime mold Dictyostelium discoideum. Am J Botany 43, 673-677.

Orlowski, M. \& Loomis, W. F. (1979). Plasma membrane proteins of Dictyostelium: the spore coat proteins. Dev Biol 71, 297-307.

Richardson, D. L. \& Loomis, W. F. (1992). Disruption of the sporulation-specific gene SpiA in Dictyostelium discoideum leads to spore instability. Genes Dev 6, 1058-1070.

Smith, E., Gooley, A. A., Hudson, G. C. \& Williams, K. L. (1989). Glycoproteins that exhibit extensive size polymorphisms in Dictyostelium discoideum. Genetics 122, 59-64.

Srinivasan, S., Alexander, H. \& Alexander, S. (1999). The prespore vesicles of Dictyostelium discoideum: Purification, characterization and developmental regulation. J Biol Chem 274, 35823-35831.

Srinivasan, S., Alexander, H. \& Alexander, S. (2000). Crossing the finish line of development: regulated secretion of Dictyostelium proteins. Trends Cell Biol 10, 215-219.

Sussman, M. (1987). Cultivation and synchronous morphogenesis of Dictyostelium under controlled experimental conditions. Methods Cell Biol 28, 9-29.

Voet, L., Krefft, M., Bruderlein, M. \& Williams, K. (1985). Flow cytometer study of anterior-like cells in Dictyostelium discoideum. J Cell Sci 75, 423-435.

Watson, N., Williams, K. L. \& Alexander, S. (1993). A developmentally regulated glycoprotein complex from Dictyostelium discoideum. J Biol Chem 268, 22634-22641.

Watson, N., McGuire, V. \& Alexander, S. (1994). The PsB glycoprotein complex is secreted as a preassembled precursor of the spore coat in Dictyostelium discoideum. J Cell Sci 107, 2567-2579.

West, C. M. \& Erdos, G. W. (1990). Formation of the Dictyostelium spore coat. Dev Genet 11, 492-506.

West, C. M., Mao, J., van der Wel, H., Erdos, G. W. \& Zhang, Y. (1996). SP75 is encoded by the DP87 gene and belongs to a family of modular Dictyostelium discoideum outer layer spore coat proteins. Microbiology 142, 2227-2243.

Yan, J. X., Gooley, A. A. \& Williams, K. L. (1997). The Dictyostelium proteome project: Studying the readout of the genome. In Dictyostelium: a Model System for Cell and Developmental Biology, pp. 455-469. Edited by Y. Maeda, K. Inouye \& I. Takeuchi. Tokyo: Universal Academy Press.

Zhang, Y. Y., Brown, R. D. \& West, C. M. (1998). Two proteins of the Dictyostelium spore coat bind to cellulose in vitro. Biochemistry 37, 10766-10779.

Zhang, Y., Zhang, P. \& West, C. M. (1999). A linking function for the cellulose-binding protein of SP85 in the spore coat of Dictyostelium discoideum. J Cell Sci 112, 4367-4377.

Received 23 December 1999; revised 11 February 2000; accepted 25 April 2000. 